\title{
A proof of Brouwer's toughness conjecture
}

\author{
Xiaofeng $\mathrm{Gu}^{*}$
}

\begin{abstract}
The toughness $t(G)$ of a connected graph $G$ is defined as $t(G)=\min \left\{\frac{|S|}{c(G-S)}\right\}$, in which the minimum is taken over all proper subsets $S \subset V(G)$ such that $c(G-S)>1$, where $c(G-S)$ denotes the number of components of $G-S$. Let $\lambda$ denote the second largest absolute eigenvalue of the adjacency matrix of a graph. For any connected $d$-regular graph $G$, it has been shown by Alon that $t(G)>\frac{1}{3}\left(\frac{d^{2}}{d \lambda+\lambda^{2}}-1\right)$, through which, Alon was able to show that for every $t$ and $g$ there are $t$-tough graphs of girth strictly greater than $g$, and thus disproved in a strong sense a conjecture of Chvátal on pancyclicity. Brouwer independently discovered a better bound $t(G)>\frac{d}{\lambda}-2$ for any connected $d$-regular graph $G$, while he also conjectured that the lower bound can be improved to $t(G) \geq \frac{d}{\lambda}-1$. We confirm this conjecture.
\end{abstract}

MSC: 05C42 05C50

Key words: toughness; eigenvalue; expander mixing lemma; pseudo-random graph

\section{The conjecture}

We use $\lambda_{i}(G)$ to denote the $i$ th largest eigenvalue of the adjacency matrix of a simple graph $G$ on $n$ vertices, for $i=1,2, \cdots, n$. By the Perron-Frobenius Theorem, $\lambda_{1}$ is always positive (unless $G$ has no edges) and $\left|\lambda_{i}\right| \leq \lambda_{1}$ for all $i \geq 2$. Let $\lambda=\max _{2 \leq i \leq n}\left|\lambda_{i}\right|=\max \left\{\left|\lambda_{2}\right|,\left|\lambda_{n}\right|\right\}$, that is, $\lambda$ is the second largest absolute eigenvalue. For a $d$-regular graph, it is well known that $\lambda_{1}=d$.

Let $c(G)$ denote the number of components of a graph $G$. The toughness $t(G)$ of a connected graph $G$ is defined as $t(G)=\min \left\{\frac{|S|}{c(G-S)}\right\}$, where the minimum is taken over all proper subsets $S \subset V(G)$ such that $c(G-S)>1$. A graph $G$ is $k$-tough if $t(G) \geq k$. Graph toughness was introduced by Chvátal [10] in 1973 and is closely related to many graph properties, including connectivity, Hamiltonicity, pancyclicity, factors, spanning trees, etc.

The study of toughness from eigenvalues was initiated by Alon [1] who showed that for any connected $d$-regular graph $G, t(G)>\frac{1}{3}\left(\frac{d^{2}}{d \lambda+\lambda^{2}}-1\right)$, through which, Alon was able to show that for every $t$ and $g$ there are $t$-tough graphs of girth strictly greater than $g$. This strengthened a result of Bauer, Van den Heuvel and Schmeichel [4] who showed the same for $g=3$, and thus disproved in

\footnotetext{
${ }^{*}$ University of West Georgia, Carrollton, GA 30118 (xgu@westga.edu). This research was partially supported by a grant from the Simons Foundation (522728, XG).
} 
a strong sense a conjecture of Chvátal [10] that there exists a constant $t_{0}$ such that every $t_{0}$-tough graph is pancyclic.

Brouwer [5] independently discovered a better bound and showed that $t(G)>\frac{d}{\lambda}-2$ for a connected $d$-regular graph $G$. He mentioned in [5] that the bound might be able to be improved a little to $\frac{d}{\lambda}-1$, and then posed the exact conjecture as an open problem in [6]. Some partial results have been provided in [8]. However, no substantial progress has been made for more than two decades. Most recently, the author improved the bound to $t(G)>\frac{d}{\lambda}-\sqrt{2}$ in [11.

Conjecture 1 (Brouwer [5, 6]). For any connected d-regular graph $G, t(G) \geq \frac{d}{\lambda}-1$.

It is mentioned by Brouwer [5] that there are infinitely many graphs $G$ such that $t(G) \leq d / \lambda$ with equality in many cases (for example, strongly regular graphs constructed in [5] and [9], and many Kneser graphs [14]). Brouwer [5] also pointed out that Conjecture 1, if true, is tight. To see sharpness, one may notice that the toughness can be arbitrarily close to 0 , while $d / \lambda \geq 1$ holds for all $d$-regular graphs. Cioabă and Wong [9] briefly described an explicit construction of such extremal graphs.

In this paper, we confirm Conjecture 1.

Theorem 1. Let $G$ be a connected d-regular graph. Then $t(G) \geq \frac{d}{\lambda}-1$.

\section{The proof}

Our main tool is the expander mixing lemma. A $d$-regular graph on $n$ vertices with the second largest absolute eigenvalue at most $\lambda$ is called an $(n, d, \lambda)$-graph. It is well known that an $(n, d, \lambda)$ graph for which $\lambda=\Theta(\sqrt{d})$ is a very good pseudo-random graph behaving, in many aspects, like a truly random graph $G(n, p)$. The quantitative definition of pseudo-random graphs was introduced by Thomason [15,16] who defined jumbled graphs.

The celebrated expander mixing lemma is usually attributed to Alon and Chung [2. This idea appeared earlier with a different form in the PhD thesis [12] of Haemers. We present this lemma in Theorem 2 (see [13] for a complete proof). A variation can be found in [3, Chapter 9] by Alon and Spencer. We refer readers to the informative survey [13] by Krivelevich and Sudakov for more about the expander mixing lemma and pseudo-random graphs. As mentioned in [13, the expander mixing lemma is a truly remarkable result, connecting edge distribution and graph spectrum, and providing a very good quantitative handle for the uniformity of edge distribution based on graph eigenvalues.

For every two subsets $A$ and $B$ of $V(G)$, let $e(A, B)$ denote the number of edges with one end in $A$ and the other one in $B$ (edges with both ends in $A \cap B$ are counted twice). We use $e(A)$ to denote the number of edges with both ends in $A$, and thus $e(A, A)=2 e(A)$. 
Theorem 2 (Expander Mixing Lemma). Let $G$ be an $(n, d, \lambda)$-graph. Then for every two subsets $A$ and $B$ of $V(G)$,

$$
\left|e(A, B)-\frac{d}{n}\right| A|| B|| \leq \lambda \sqrt{|A||B|\left(1-\frac{|A|}{n}\right)\left(1-\frac{|B|}{n}\right)} .
$$

In particular,

$$
\left.\left|e(A)-\frac{d}{2 n}\right| A\right|^{2}\left|\leq \frac{\lambda}{2}\right| A \mid\left(1-\frac{|A|}{n}\right) .
$$

The following lemma has been proved and used by Brouwer and Haemers [7]. For the sake of completeness, we include a short proof here.

Lemma 3 ([7]). Let $x_{1}, \cdots, x_{c}$ be positive integers such that $\sum_{i=1}^{c} x_{i} \leq 2 c-1$. Then for every integer $\ell$ with $0 \leq \ell \leq \sum_{i=1}^{c} x_{i}$, there exists an $I \subset\{1, \cdots, c\}$ such that $\sum_{i \in I} x_{i}=\ell$.

Proof. The proof provided by Brouwer and Haemers [7] goes by induction on $c$. The case $c=1$ is trivial. Let $c \geq 2$ and assume that $x_{1} \leq \cdots \leq x_{c}$. Suppose it is true for $c-1$ integers. Let $\ell^{\prime}=\ell$ if $\ell \leq c-1$ and $\ell^{\prime}=\ell-x_{c}$ otherwise. Since $c-1 \leq \sum_{i=1}^{c-1} x_{i}=\sum_{i=1}^{c} x_{i}-x_{c} \leq 2(c-1)-1$, by inductive hypothesis, there exists an $I^{\prime} \subset\{1, \cdots, c-1\}$ such that $\sum_{i \in I^{\prime}} x_{i}=\ell^{\prime}$. Let $I=I^{\prime}$ or $I=I^{\prime} \cup\{c\}$, as desired.

Now we are ready to present the proof of the main theorem.

Proof of Theorem 1. Suppose to the contrary that

$$
t(G)<\frac{d}{\lambda}-1
$$

By definition, suppose $S$ is a subset of $V(G)$ such that $\frac{|S|}{c(G-S)}=t(G)$. Let $B=V(G-S)$. Denote $|V(G)|=n, c(G-S)=c$ and $t(G)=t$. Then $|S|=t c$ and so $|B|=n-t c$.

First we show that

$$
c \leq \frac{\lambda n}{d+\lambda} .
$$

In fact, (4) can be obtained from the well-known Hoffman ratio bound. Here we give a direct proof. To see this, let $U$ be a set of vertices that consists of exactly one vertex from each component of $G-S$. Then $|U|=c$ and $e(U)=0$. By (2), $\left.\left|e(U)-\frac{d}{2 n}\right| U\right|^{2}\left|\leq \frac{\lambda}{2}\right| U \mid\left(1-\frac{|U|}{n}\right)$, and thus $\frac{d}{n}|U| \leq \lambda\left(1-\frac{|U|}{n}\right)$, which implies that $c=|U| \leq \frac{\lambda n}{d+\lambda}$.

If $|B| \leq \frac{2 \lambda n}{d+\lambda}$, then $t c=n-|B| \geq \frac{(d-\lambda) n}{d+\lambda}$. Together with (44), we have

$$
t=(n-|B|) / c \geq \frac{(d-\lambda) n}{d+\lambda} \cdot \frac{d+\lambda}{\lambda n}=\frac{d-\lambda}{\lambda}=\frac{d}{\lambda}-1,
$$

contrary to (3). Thus, we may assume that

$$
|B|>\frac{2 \lambda n}{d+\lambda},
$$


By (44) and (5), we have $|B|>2 c$, that is

$$
|B| \geq 2 c+1
$$

Let $V_{1}, \cdots, V_{c}$ denote the vertex sets of the $c$ components of $G-S$. Without loss of generality, we may assume that $\left|V_{1}\right| \leq \cdots \leq\left|V_{c}\right|$.

Claim 1: $\sum_{i=1}^{c-1}\left|V_{i}\right| \geq c$.

Proof of Claim 1: Otherwise, $\sum_{i=1}^{c-1}\left|V_{i}\right|=c-1$, that is, each $V_{i}$ is a single vertex for $i=1, \cdots, c-1$. Let $A=\cup_{i=1}^{c-1} V_{i}$ and thus $|A|=c-1$ and $e(A, B)=0$. By (1),

$$
\frac{d}{n}|A||B| \leq \lambda \sqrt{|A||B|\left(1-\frac{|A|}{n}\right)\left(1-\frac{|B|}{n}\right)}<\lambda \sqrt{|A||B|\left(1-\frac{|B|}{n}\right)},
$$

and so

$$
\left(\frac{d}{n}|A||B|\right)^{2}<\lambda^{2}|A||B|\left(1-\frac{|B|}{n}\right)
$$

which implies that

$$
\frac{d^{2}|A||B|}{\lambda^{2} n}<n-|B|=t c
$$

Thus

$$
t>\frac{|A|}{c} \cdot \frac{d^{2}}{\lambda^{2}} \cdot \frac{|B|}{n}=\frac{c-1}{c} \cdot \frac{d^{2}}{\lambda^{2}} \cdot \frac{|B|}{n}=\left(1-\frac{1}{c}\right) \cdot \frac{d^{2}}{\lambda^{2}} \cdot \frac{|B|}{n} \geq \frac{d^{2}}{2 \lambda^{2}} \cdot \frac{|B|}{n} .
$$

By (5), $\frac{|B|}{n}>\frac{2 \lambda}{d+\lambda}$ and we have

$$
t>\frac{d^{2}}{2 \lambda^{2}} \cdot \frac{2 \lambda}{d+\lambda}=\frac{d^{2}}{\lambda(d+\lambda)}=\frac{d^{2}-\lambda^{2}+\lambda^{2}}{\lambda(d+\lambda)}=\frac{d^{2}-\lambda^{2}}{\lambda(d+\lambda)}+\frac{\lambda^{2}}{\lambda(d+\lambda)}>\frac{d}{\lambda}-1,
$$

contrary to (3). This completes the proof of Claim 1 .

Claim 2: $V_{1}, \cdots, V_{c}$ can be partitioned into two disjoint sets $X$ and $Y$ such that $e(X, Y)=0$, $|X| \geq c$ and $|Y| \geq c$.

Proof of Claim 2: If $\left|V_{c}\right| \geq c$, then let $X=\cup_{i=1}^{c-1} V_{i}$ and $Y=B-X=V_{c}$. Then $|Y| \geq c$ and by Claim $1,|X| \geq c$. Thus we may assume that $\left|V_{c}\right| \leq c-1$. We also notice that $\left|V_{c}\right| \geq 3$, for otherwise, $|B|=\sum_{i=1}^{c}\left|V_{i}\right| \leq 2 c$, contradicting ([6). Thus $3 \leq\left|V_{c}\right| \leq c-1$. Let $\ell=c-\left|V_{c}\right|$. Then $1 \leq \ell \leq c-3$.

If $\sum_{i=1}^{c-1}\left|V_{i}\right| \leq 2(c-1)-1=2 c-3$, then by Lemma 3, there exists an $I \subset\{1, \cdots, c-1\}$ such that $\sum_{i \in I}\left|V_{i}\right|=\ell$. Let $X=\cup_{i \in I} V_{i} \cup V_{c}$ and $Y=B-X$. Clearly $|X|=\ell+\left|V_{c}\right|=c$ and $|Y|=|B|-|X| \geq c+1$.

If $\sum_{i=1}^{c-1}\left|V_{i}\right|>2(c-1)-1=2 c-3$, then let $V_{i}^{\prime}$ be a nonempty subset of $V_{i}$ for each $i=1,2, \cdots c-1$ such that $\sum_{i=1}^{c-1}\left|V_{i}^{\prime}\right|=2 c-3$. We can easily do it by removing vertices from some $V_{i}$ 's one by one until the total number of remaining vertices is exactly $2 c-3$ (keep at least one vertex in each subset). By Lemma 3 , there exists an $I \subset\{1, \cdots, c-1\}$ such that $\sum_{i \in I}\left|V_{i}^{\prime}\right|=\ell$. Then 
$\sum_{i \notin I, i<c}\left|V_{i}^{\prime}\right|=(2 c-3)-\ell \geq(2 c-3)-(c-3)=c$. Let $X=\cup_{i \in I} V_{i} \cup V_{c}$ and $Y=B-X=\cup_{i \notin I, i<c} V_{i}$. Clearly $|X| \geq \ell+\left|V_{c}\right|=c$ and $|Y|=\sum_{i \notin I, i<c}\left|V_{i}\right| \geq \sum_{i \notin I, i<c}\left|V_{i}^{\prime}\right| \geq c$. This completes the proof of Claim 2.

By Claim 2, $V_{1}, \cdots, V_{c}$ can be partitioned into two disjoint sets $X$ and $Y$ such that $e(X, Y)=0$, $|X| \geq c$ and $|Y| \geq c$.

Since $e(X, Y)=0$, by (11), $\frac{d}{n}|X||Y| \leq \lambda \sqrt{|X||Y|\left(1-\frac{|X|}{n}\right)\left(1-\frac{|Y|}{n}\right)}$, and so

$$
d^{2}|X|^{2}|Y|^{2} \leq \lambda^{2}|X||Y|(n-|X|)(n-|Y|),
$$

which implies that

$$
|X||Y| \leq \frac{\lambda^{2}}{d^{2}}(n-|X|)(n-|Y|) .
$$

Without loss of generality, we may assume that $|X| \leq|Y|$. By (7), we have

$$
|X|^{2} \leq|X| \cdot|Y| \leq \frac{\lambda^{2}}{d^{2}}(n-|X|)(n-|Y|) \leq \frac{\lambda^{2}}{d^{2}}(n-|X|)^{2}
$$

that is

$$
|X| \leq \frac{\lambda}{d}(n-|X|)
$$

and hence

$$
|X| \leq \frac{\lambda n}{d+\lambda}
$$

Also, since $|Y|=n-|S|-|X|$, by (7) again, we have

$$
|X|(n-|S|-|X|)=|X| \cdot|Y| \leq \frac{\lambda^{2}}{d^{2}}(n-|X|)(n-|Y|)=\frac{\lambda^{2}}{d^{2}}(n-|X|)(|S|+|X|),
$$

which implies that

$$
|X| n \leq\left(\frac{\lambda^{2}}{d^{2}}(n-|X|)+|X|\right)(|S|+|X|)=\left(\frac{\lambda^{2}}{d^{2}} n+\frac{d^{2}-\lambda^{2}}{d^{2}}|X|\right)(|S|+|X|) .
$$

By (8), we have

$$
\frac{d^{2}-\lambda^{2}}{d^{2}}|X| \leq \frac{d^{2}-\lambda^{2}}{d^{2}} \cdot \frac{\lambda n}{d+\lambda}=\frac{d \lambda-\lambda^{2}}{d^{2}} n
$$

plugging in (9),

$$
|X| n \leq\left(\frac{\lambda^{2}}{d^{2}} n+\frac{d \lambda-\lambda^{2}}{d^{2}} n\right)(|S|+|X|)=\frac{\lambda n}{d}(|S|+|X|),
$$

and we have

$$
|X| \leq \frac{\lambda}{d}(|S|+|X|)
$$

Hence

$$
t c=|S| \geq\left(\frac{d}{\lambda}-1\right)|X|
$$

implying that

$$
t \geq\left(\frac{d}{\lambda}-1\right) \cdot \frac{|X|}{c} \geq \frac{d}{\lambda}-1
$$

completing the proof of the theorem. 


\section{References}

[1] N. Alon, Tough Ramsey graphs without short cycles, J. Algebraic Combin. 4 (1995), 189-195.

[2] N. Alon and F. R. K. Chung, Explicit construction of linear sized tolerant networks, Discrete Math. 72 (1988), 15-19.

[3] N. Alon and J. H. Spencer, The Probabilistic Method, Wiley, 2016.

[4] D. Bauer, J. van den Heuvel and E. Schmeichel, Toughness and triangle-free graphs, J. Combin. Theory Ser. B 65 (1995), 208-221.

[5] A. E. Brouwer, Toughness and spectrum of a graph, Linear Algebra Appl. 226/228 (1995), $267-271$.

[6] A. E. Brouwer, Spectrum and connectivity of graphs, CWI Quarterly 9 (1996), 37-40.

[7] A. E. Brouwer and W. H. Haemers, Eigenvalues and perfect matchings, Linear Algebra Appl. 395 (2005), 155-162.

[8] S. M. Cioabă and X. Gu, Connectivity, toughness, spanning trees of bounded degrees, and spectrum of regular graphs, Czechoslovak Math. J. 66 (2016), 913-924.

[9] S. M. Cioabă and W. Wong, The spectrum and toughness of regular graphs. Discrete Appl. Math. 176 (2014), 43-52.

[10] V. Chvátal, Tough graphs and hamiltonian circuits, Discrete Math. 5 (1973), 215-228.

[11] X. Gu, Toughness in pseudo-random graphs, European J. Combin., 92 (2021), 103255.

[12] W. H. Haemers, Eigenvalue techniques in design and graph theory, PhD thesis, 1979.

[13] M. Krivelevich and B. Sudakov, Pseudo-random graphs, More sets, graphs and numbers, Bolyai Soc. Math. Stud., 15, Springer, Berlin, 2006, 199-262.

[14] D. Park, A. Ostuni, N. Hayes, A. Banerjee, T. Wakhare, W. Wong and S. Cioabă, The toughness of Kneser graphs, arXiv:2008.08183 [math.CO].

[15] A. Thomason, Pseudo-random graphs, in: Proceedings of Random Graphs, Poznań 1985, M. Karoński, ed., Annals of Discrete Math. 33 (1987), 307-331.

[16] A. Thomason, Random graphs, strongly regular graphs and pseudo-random graphs, Surveys in Combinatorics, 1987, C. Whitehead, ed., LMS Lecture Note Series 123 (1987), 173-195. 